\title{
A non-linear and stochastic response surface method for Bayesian estimation of uncertainty in soil moisture simulation from a land surface model
}

\author{
F. Hossain, E. N. Anagnostou, and K.-H. Lee \\ University of Connecticut, Department of Civil and Environmental Engineering, Storrs, Connecticut, USA \\ Received: 16 March 2004 - Revised: 30 June 2004 - Accepted: 12 July 2004 - Published: 24 September 2004 \\ Part of Special Issue "Nonlinear deterministic dynamics in hydrologic systems: present activities and future challenges"
}

\begin{abstract}
This study presents a simple and efficient scheme for Bayesian estimation of uncertainty in soil moisture simulation by a Land Surface Model (LSM). The scheme is assessed within a Monte Carlo (MC) simulation framework based on the Generalized Likelihood Uncertainty Estimation (GLUE) methodology. A primary limitation of using the GLUE method is the prohibitive computational burden imposed by uniform random sampling of the model's parameter distributions. Sampling is improved in the proposed scheme by stochastic modeling of the parameters' response surface that recognizes the non-linear deterministic behavior between soil moisture and land surface parameters. Uncertainty in soil moisture simulation (model output) is approximated through a Hermite polynomial chaos expansion of normal random variables that represent the model's parameter (model input) uncertainty. The unknown coefficients of the polynomial are calculated using limited number of model simulation runs. The calibrated polynomial is then used as a fast-running proxy to the slower-running LSM to predict the degree of representativeness of a randomly sampled model parameter set. An evaluation of the scheme's efficiency in sampling is made through comparison with the fully random MC sampling (the norm for GLUE) and the nearest-neighborhood sampling technique. The scheme was able to reduce computational burden of random MC sampling for GLUE in the ranges of $10 \%-70 \%$. The scheme was also found to be about $10 \%$ more efficient than the nearestneighborhood sampling method in predicting a sampled parameter set's degree of representativeness. The GLUE based on the proposed sampling scheme did not alter the essential features of the uncertainty structure in soil moisture simulation. The scheme can potentially make GLUE uncertainty estimation for any LSM more efficient as it does not impose any additional structural or distributional assumptions.
\end{abstract}

Correspondence to: E. N. Anagnostou

(manos@engr.uconn.edu)

\section{Introduction}

In hydrology, uncertainty estimation techniques that are based on fully random Monte Carlo (MC) sampling of probability distributions are usually considered the preferred method due to their lack of restrictive assumptions, completeness in sampling the error structure of the random variables, and the increasing availability of computational resources (Beven and Freer, 2001; Beck, 1987; Kremer, 1983). MC sampling can also bypass several limitations of analytical techniques (Bras and Rodriguez-Iturbe, 1993). An uncertainty estimation technique called Generalized Likelihood Uncertainty Estimation (GLUE) (Beven and Binley, 1992) is one such MC based tool that can be employed to assess an environmental model's predictive uncertainty. This method extends the type of Generalized Sensitivity Analysis (GSA) of Spear and Hornberger (1980) by evaluating the simulation results for each randomly sampled model parameter set against some observed data through a likelihood value. Because its structure is rooted in Bayesian theory, GLUE also allows blending of prior and current information for improved a posteriori inferences. While GLUE is not the only uncertainty assessment tool currently available (Misirli et al., 2003; Thiemann et al., 2001; Tyagi and Haan, 2001; Krzysztofowicz, 2000; Young and Beven, 1994), the simplicity of the theory behind the technique is what makes it convenient and very easy to implement (Beven and Freer, 2001). GLUE has therefore found extensive application in the assessment of predictive uncertainty of many hydrologic variables like streamflow, flood inundation, ground water flow, land surface fluxes, etc. (Schulz and Beven, 2003; Christaens and Feyen, 2002; Beven and Freer, 2001; Schulz et al., 2001; Romanowicz and Beven, 1998; Franks et al., 1998; Franks and Beven, 1997; Freer et al., 1996; among many others). Recently, the GLUE technique has also proved to be a powerful tool in understanding the implications of remotely sensed rainfall error adjustment on flood prediction uncertainty (Hossain et al., 2004). 
However, the GLUE method has a major drawback. It requires analysis of multiple simulation scenarios based on uniform random sampling of the model parameter hyperspace. This requirement can be computationally prohibitive for physically complex models that are slow-running (Bates and Campbell, 2001; Beven and Binley, 1992). Beven and Binley (1992) have argued in detail that the assumption of uniform distribution is unlikely to prove critical for GLUE. Freer et al. (1996) have further justified uniform sampling because it makes the GLUE procedure simple to implement and avoids the necessity to sample from some multivariate set of correlated distributions which is often very difficult to justify from observed data. Nevertheless, the drawback of uniformity assumption in GLUE magnifies tremendously for physically complex Land Surface Models (LSM) that simultaneously balance water and energy budget across the land surface. Thus, GLUE application for Bayesian estimation of uncertainty in land surface-atmosphere flux predictions has so far been limited to relatively simpler conceptualizations of soil-vegetation-atmosphere transfer (SVAT) schemes (e.g. Schulz and Beven, 2003; Schulz et al., 2001; Franks et al., 1998; Franks and Beven, 1997). A more realistic Bayesian assessment of uncertainty requires the application of GLUE to physically complex operational LSMs such as Common Land Model (CLM; Dai et al., 2003), NOAH-LSM (Pan and Mahrt, 1987), BATS (Dickinson et al., 1986) or SiB (Sellers et al., 1986). Uncertainty assessment of these models are important because, despite their physical complexity, they nevertheless suffer from parameter equifinality where a wide range of parameter sets exhibit equally acceptable simulations against data available.

In the last decade, researchers have strived to develop numerical schemes for efficient sensitivity analyses of LSM parameters. Henderson-Sellers (1993) proposed a Factorial Assessment (FA) of sensitivity of model parameters that incorporates the multifactor interactions and tries to avoid the potential weakness of the classical sensitivity analyses of perturbing one parameter at a time. However, the FA method suffers from the following limiting requirements: 1) prior knowledge of parameter variances; and 2) large number of model perturbations (Gao et al., 1996). Collins and Avissar (1994) proposed a Fourier Amplitude Sensitivity Test (FAST) for land surface parameters. This method also has drawbacks similar to the FA method with the additional requirement that parameters be physically uncorrelated. Gao et al. (1996) summarized that there was no perfect method for characterizing parameter uncertainty of land surface systems and proposed a special form of the classical stand-alone sensitivity analyses for land-surface schemes. Our qualitative assessment of the techniques reported in literature and alluded herein indicates that none of them are pertinent to GLUE for making uncertainty estimation of LSMs computationally more efficient.

In recognition of the uncertainty due to input land surface parameters and the ease of implementation of the GLUE method, there is a need to develop a parameter sampling technique that can make the application of GLUE more efficient for LSMs. Such a technique should not impose additional structural or distributional assumptions that may otherwise compromise the inherent simplicity of the GLUE method. Kuczera and Parent (1998) and Bates and Campbell (2001) have already explored the use of Markov Chain Monte Carlo (MCMC) methods for more efficient parameter uncertainty analyses. Bates and Campbell (2001) however reported that MCMC methods cannot be used as a black box - considerable care is required in its implementation when models have large number of parameters. A further criticism made by Beven and Freer (2001) was that MCMC methods can rarely be useful in making considerable savings in computing time when the model response surface with respect to parameters is not well defined and has the presence of multiple local maxima or plateau. Christaens and Feyen (2002) employed the Latin Hypercube Sampling (LHS) method to accelerate parameter sampling for the MIKE-SHE hydrologic model. However, LHS is based on the assumption of monotonicity of model output in terms of input parameters, in order to be unconditionally guaranteed of accuracy with an order of magnitude fewer runs than uniform random sampling (McKay et al., 1979; Iman et al., 1981). Recent study by Hossain et al. $(2004 a)^{1}$ has clearly shown that the use of LHS method is not always effective and that it requires care in planning an effective sampling strategy. Consequently this study is motivated by the need to develop a simple but efficient parameter sampling technique that can make GLUE computationally more efficient for slow-running LSMs.

In the current state of the art, GLUE for such models would require an interpolator for the model parameter-output response surface. This interpolator could then act as a fastrunning proxy to the slow running model and potentially identify the regions of high likelihood values (i.e. regions of high degree of representativeness of the hydrologic system) on the parameter-output response surface. In this study we have chosen to develop a stochastic interpolator based on the "Theory of Homogeneous Chaos" (Wiener, 1938) (hereafter called "interpolator"). We do not demonstrate the presence or absence of chaotic behavior of simulations in this study. However, we are encouraged by the recent well-documented discovery of chaos in hydrologic systems (Sivakumar et al., 2001a and 2001b; Sivakumar, 2000; Jayawardena and Lai, 1994; Rodriguez-Iturbe et al., 1991). Essential concepts of the interpolator are inferred from an uncertainty estimation tool originally developed by Isukapalli et al. (2000). However, the critical evaluation presented herein of the interpolator within the GLUE framework for improving parameter sampling is considered a relatively unexplored topic. In this study we make an evaluation of the interpolator on a different surface hydrologic variable - soil moisture - which is simulated by the physically-based NOAH-LSM (Pan and Mahrt, 1987). The interpolator is also compared with the

\footnotetext{
${ }^{1}$ Hossain, F., Anagnostou, E. N., and Bagtzoglou, A. C.: On Latin Hypercube Sampling for Efficient Uncertainty Estimation of Satellite-derived runoff predictions. J. of Hydrology, in review, 2004.
} 

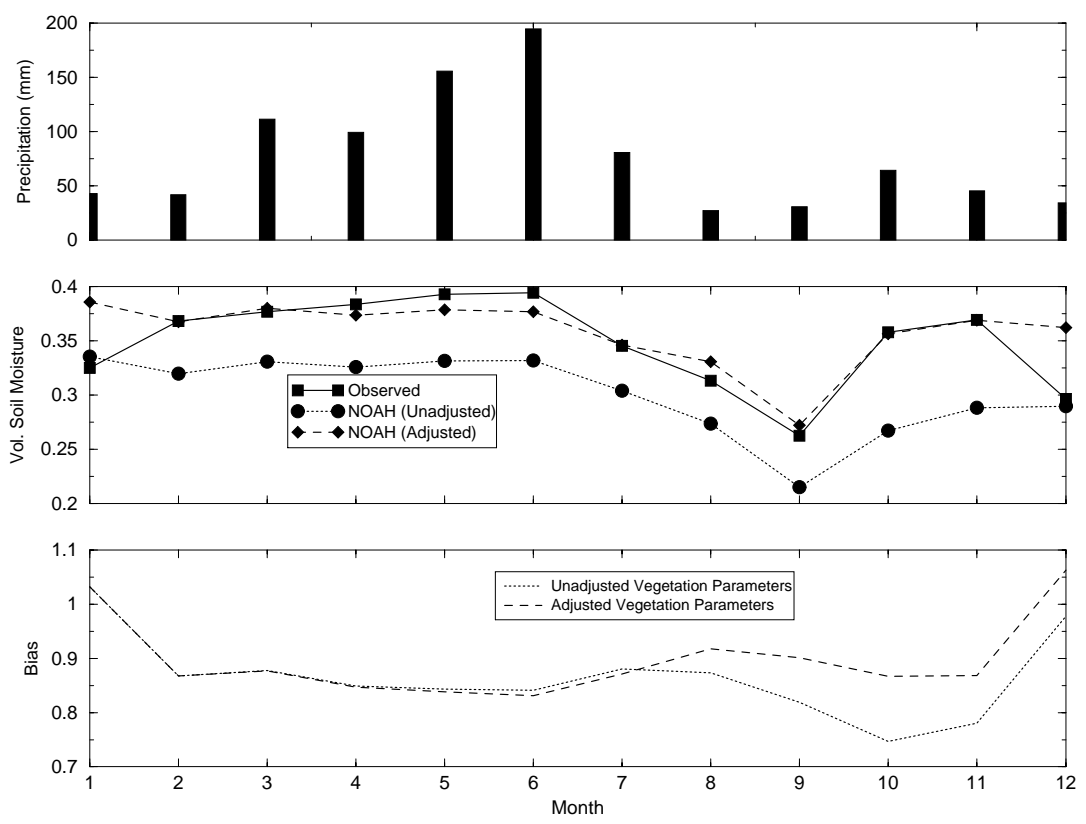

Fig. 1. NOAH-LSM vegetation parameter and bias adjustment for soil moisture simulation. Upper panel - monthly accumulated precipitation (mm) for 1998. Middle panel - observed soil moisture measurements (mean monthly) at $5 \mathrm{~cm}$ depth compared with simulations with adjustments and no adjustments. Lower panel - the monthly multiplicative bias in simulation with adjusted and unadjusted vegetation parameters. Effective study period was 1 March - 30 November 1998.

fully random MC sampling technique (the norm for GLUE) and the nearest-neighborhood parameter sampling technique originally proposed by Beven and Binley (1992).

The study is organized in the following manner. In Sect. 2, a brief description of the study region and data are provided. Section 3 describes the LSM part a and its readjustment part $\mathrm{b}$ that were found necessary to make the model representative of the study region. In Sect. 4, we describe the GLUE method based on fully random uniform parameter sampling. Section 5 provides description of the algorithm for the interpolator for parameter sampling. Section 6 describes the simulation framework for assessment of the interpolator. Section 7 discusses the results, while Sect. 8 presents the conclusions and further extensions that can potentially extend the capabilities of the interpolator.

\section{Study region and data}

Our study region was Northern Illinois (USA) in a farmland in Champaign located $40.01^{\circ} \mathrm{N}$ and $88.37^{\circ} \mathrm{W}$. The site characteristics were typical of those found throughout Midwestern US with most of the land in agricultural production. The soil was silt loam with a bulk density of $1.5 \mathrm{gm} / \mathrm{cm}^{3}$. The year under study was 1998 when soybeans were planted in the farm. Atmospheric and radiation forcing data from a flux measuring system installed in the farm was recorded every $30 \mathrm{~min}$ for that year. The major atmospheric data comprised precipitation, temperature, humidity, surface pressure and wind. The radiation forcing data pertained to downward solar (short-wave) and downward long-wave radiation flux measurements. This data is public domain and available as part of standardized testing protocols for simulation codes of the NOAH-LSM (discussed next). To reduce the impact of snow and sensitivity to initial conditions in our study, we chose an effective study period ranging from 1 March 30 November 1998. For more information on the study region and data measurement protocols the reader is referred to the User's Guide, Public Release Version 2.5 available at $\mathrm{ftp} / / / \mathrm{ftp}$.emc.noaa.gov/mmb/gcp/ldas/noahlsm/ver_2.5.

\section{The land surface model}

\subsection{Model description}

The LSM used in this study was NOAH-LSM (also known as The Community NOAH-LSM) (Pan and Mahrt, 1987). We chose NOAH-LSM as it is a popular operational model and insights into this study could prove beneficial in understanding the utility of the proposed sampling technique for uncertainty prediction of land surface variables in general. This LSM is a stand-alone, uncoupled, 1-D column version used to execute single-site land surface simulations. In this traditional 1-D uncoupled mode, near surface atmospheric and radiation forcing data are required as input forcing. NOAHLSM simulates soil moisture (both liquid and frozen), soil temperature, snow pack, depth, snow pack water equivalent, canopy water content and the energy and water flux terms in terms of the surface energy balance and surface water balance. A four-layer soil configuration (comprising a total depth of $2 \mathrm{~m}$ ) is adopted in the NOAH-LSM for captur- 


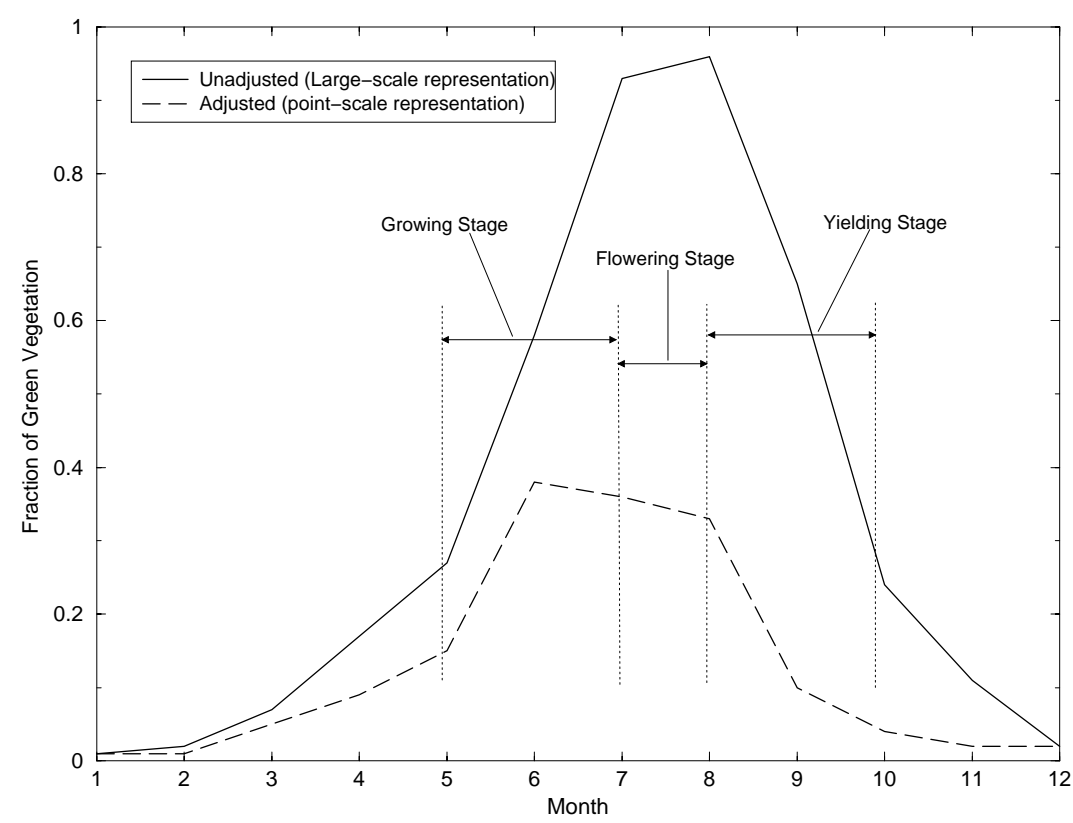

Fig. 2. Readjustment of monthly Fraction of Vegetation parameters for NOAH-LSM to make them more representative of the study region and point-scale simulation of soil moisture for the upper $5 \mathrm{~cm}$ layer. Unadjusted parameters are derived from Normalized Difference Vegetation Index (NDVI; Gutman and Ignatov, 1998). The growth sequence applies for soybeans that were planted during 1998.

ing daily, weekly and seasonal evolution of soil moisture and mitigating possible truncation error in discretization (Sridhar et al., 2002). The lower $1 \mathrm{~m}$ acts as gravity drainage at the bottom, and the upper 1meter of soil serves as root zone depth. Since this study concerns the assessment of a parameter sampling technique, we have considered soil moisture observations and simulations only at the $5 \mathrm{~cm}$ depth for the sake of simplicity. For more details on the physical description of the model, one may refer to Sridhar et al. (2002).

\subsection{Model readjustment}

Our preliminary investigation with NOAH-LSM revealed significant underestimation of soil moisture simulation at the $5 \mathrm{~cm}$ depth. This thereby indicated an overestimation of Evapotranspiration (ET) process that magnified further during the soybean growing season (see Fig. 1, lower panel). We therefore found it necessary to adjust some of the NOAHLSM vegetation parameters to make the model more representative of the point-scale soil moisture flux simulations at the farm. We reduced the number of root layers from 3 (100 cm of deep roots) to 2 ( $40 \mathrm{~cm}$ of deep roots). This reduction was justified for our study period, as soybeans do not typically grow roots beyond $30 \mathrm{~cm}$ depth (Norman, 1978; Liu, 1997). We found Leaf Area Index (LAI) to be an insensitive parameter to the bias in soil moisture simulation. We further hypothesized that a typical soybeans lateral spacing of $80 \mathrm{~cm}$ (inferred from: Norman, 1978) should not yield the fraction of green vegetation greater than 0.5 during the growing months. The vegetation fraction parameters used in LSMs are derived from the NDVI (Normalized Difference Vegetation Index) proposed by Gutman and Ig- natov (1998). Because NDVI as derived from the NOAA AVHRR are typically representative for the $15 \times 15 \mathrm{~km}^{2}$ resolution (see Gutman and Ignatov, 1998), we argue that they may require minor adjustment for the point scale study conducted herein. The use of high resolution LANDSAT data $(30 \mathrm{~m})$ could perhaps address this limitation. However, the non-availability of such higher resolution data prompted us to assume an adjusted set of fraction of vegetation parameter for a 1-D (point) investigation scenario. We argue that this assumption is acceptable as the objective of this study is confined to the exploration of sampling efficiency of our proposed scheme. Based on knowledge of the soybean growth sequence (i.e. plant in May; flower in July and harvest in October) (Liu, 1997), we adjusted the vegetation fraction parameters as shown in Fig. 2. It is seen that the bias is now reduced after this adjustment for the growing season (MayJuly). The mean multiplicative bias (ratio of simulated to observed) for the effective study period (1 March - 30 November 1998) was found to be 0.868 (Fig. 1, lower panel). We therefore applied a final multiplicative bias adjustment factor to the NOAH-LSM soil moisture simulations of 1.15 (i.e. $1 / 0.868$ ). The effect of bias adjustment after the vegetation parameter fine-tuning is shown to improve simulations significantly (see Fig. 1, middle panel, dashed line).

\subsection{Model parameter uncertainty}

NOAH-LSM parameter uncertainty was accounted for the following five soil hydraulic parameters that we considered most sensitive to soil moisture simulation: 1) maximum volumetric soil moisture content (porosity) (SMCMAX, $\left.\mathrm{m}^{3} / \mathrm{m}^{3}\right) ; 2$ ) saturated matric potential (PSISAT, $\left.\mathrm{m}\right)(3)$ 
Table 1. Uncertainty ranges for soil hydraulic parameters of NOAH-LSM.

\begin{tabular}{lccc}
\hline Parameter & Minimum value & Maximum value & Sampling strategy \\
\hline 1. SMCMAX $\left(\mathrm{m}^{3} / \mathrm{m}^{3}\right)$ & 0.05 & 0.50 & Uniform \\
2. PSISAT $(\mathrm{m})$ & 0.01 & 0.65 & Uniform \\
3. SATDK $(\mathrm{m} / \mathrm{s})$ & $1.00 \times 10^{-6}$ & $1.77 \times 10^{-4}$ & Log (uniform) \\
4. BB & 2.00 & 15.00 & Uniform \\
5. SMCWLT $\left(\mathrm{m}^{3} / \mathrm{m}^{3}\right)$ & 0.01 & 0.20 & Uniform \\
\hline
\end{tabular}

saturated hydraulic conductivity $\mathrm{K}$ (SATDK, $\mathrm{m} \mathrm{s}^{-1}$ ); 3) parameter 'B' of soil-water retention model of Clapp and Hornberger (1978) (BB); and (4) soil moisture wilting point at which ET ceases (SMCWLT, $\mathrm{m}^{3} / \mathrm{m}^{3}$ ). The parameter uncertainty ranges are shown in Table 1 and were based on the empirical study of Clapp and Hornberger (1978) and the sampling requirements of GLUE (Beven and Binley, 1992) (discussed next).

\section{The GLUE methodology}

GLUE is based on MC simulation: a large number of model runs are made, each with random parameter values selected from uniform probability distributions for each parameter. The acceptability of each run is assessed by comparing predicted to observed hydrologic measurement through some chosen likelihood measure. Runs that achieve a likelihood below a certain threshold may then be rejected as nonbehavioral. The likelihoods of these non-behavioral parameters are set to zero and are thereby removed from the subsequent analysis. Following the rejection of non-behavioral runs, the likelihood weights of the retained (i.e. behavioral) runs are rescaled so that their cumulative total is one (Freer et al., 1996). In this study the GLUE method was applied to uncertainty estimation of soil moisture simulation by NOAHLSM at the $5 \mathrm{~cm}$ depth. Thus at each time step (at 30 minute intervals), the predicted soil moisture from the behavioral runs are likelihood weighted and ranked to form a cumulative distribution of soil moisture simulation from which chosen quantiles can be selected to represent model uncertainty. While GLUE is based on a Bayesian conditioning approach, the likelihood measure is achieved through a goodness of fit criterion as a substitute for a more traditional likelihood function. We have considered two specific likelihood measures in this study: 1) the classical index of efficiency, $E_{\mathrm{NS}}$ (Nash and Sutcliffe, 1970) (Eq. 1), and 2) the exponential index of efficiency, $E_{\mathrm{EXP}}$ (Eq. 2).

$$
\begin{aligned}
& E_{\mathrm{NS}}=\left[1-\frac{\sigma_{e}^{2}}{\sigma_{\mathrm{obs}}^{2}}\right] \\
& E_{\mathrm{EXP}}=\exp \left[\frac{-\sigma_{e}^{2}}{\sigma_{\mathrm{obs}}^{2}}\right],
\end{aligned}
$$

where $\sigma_{e}$ is the variance of errors and $\sigma_{\mathrm{obs}}$, the variance of observations. These two likelihood measures are consistent with the requirements of the GLUE method, as both increase monotonically with the similarity of behavior. The purpose of using two different likelihood measures was to demonstrate that the applicability of the interpolator was not sensitive to the subjective choice.

Now, to implement the GLUE methodology, each parameter of NOAH-LSM was specified a range of possible values shown earlier in Table 1. Constant (calibrated) values for all other NOAH-LSM parameters were used. Model predictions of soil moisture were carried out, and the model likelihood measure was calculated using the efficiency indices of Eqs. (1) and (2). From the specified parameter ranges, MC simulations were conducted that allowed the selection of a large number of behavioral parameter sets characterized by a simulation efficiency index value greater than an assigned minimum threshold value. For further details on GLUE implementation, one is referred to Beven and Binley (1992), Freer et al. (1996) and Beven and Freer (2001).

\section{Algorithm of the inperpolator}

The principle of the interpolator is founded on the "Theory of Homogeneous Chaos" (Wiener, 1938). Wiener (1938) has shown that if deterministic dynamical model is highly nonlinear (with a tendency to exhibit chaotic behavior), then it is possible to approximate both inputs and outputs (treated here as random processes) of the uncertain model through series expansion of standard random variables using Hermite Polynomials. Although the presence of chaotic behavior in the hydrologic system under study is not addressed herein, recent literature supports the wisdom of choosing the "Theory of Homogeneous Chaos" as a basis for formulation of the interpolator (Sivakumar, 2000; Sivakumar et al., 2001a, b; Rodriguez-Iturbe et al., 1991). Rodriguez-Iturbe et al. (1991) has demonstrated chaotic behavior of soil moisture dynamics at seasonal time scales. Since our effective study period was seasonal (from March to November 1998), this observation by Rodriguez-Iturbe et al. (1991) therefore justifies the use of a chaotic approach for our methodology. Furthermore, the requirement of multiple ordinary non-linear differential equations as the necessary condition for chaotic behavior in soil moisture dynamics has also been noted by Rodriguez-Iturbe 
Define uniform distribution of model parameters Transform the parameters from srvs $\mathrm{N} \sim(0,1)$ to uniform parameters (Eqs. 3 and 4)

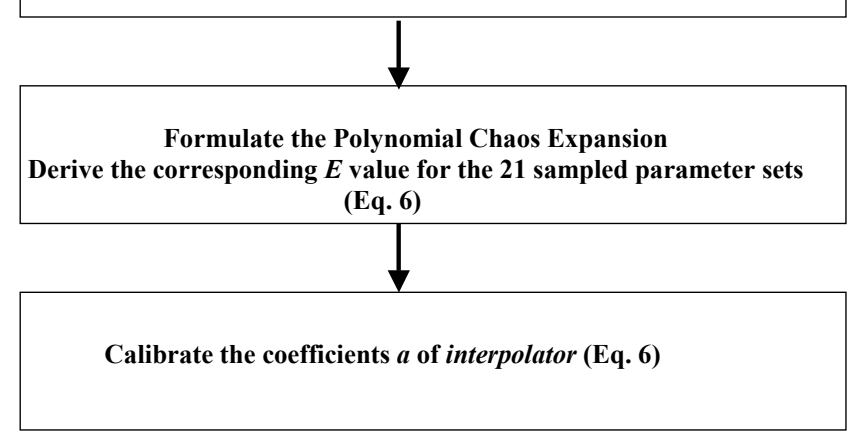

Fig. 3. Flow-chart for the algorithm of the interpolator.

et al. (1991). The physical formulation of NOAH-LSM indicates that there are sufficient physical sub-models linking the 5 soil hydraulic parameters (of Table 1) to intuitively expect a chaotic behavior relationship (between soil moisture prediction the hydraulic parameters). These notable sub-models are as follows: 1) The prognostic equation for volumetric soil water content (Richards Equation) (Sridhar et al., 2002); 2) The diffusion equation for soil temperature (Sridhar et al., 2002); 3) The Penman-based energy balance approach for potential evaporation (Sridhar et al., 2002); and, 4) The Mahrt and Ek (1984) formulation of surface skin temperature. There are three major steps involved in the algorithm formulation of this interpolator. We describe these steps below. For more details the reader is referred to Isukapalli and Georogopolous (1999) and Isukapalli et al. (2000).

\subsection{Step one: transformation of parameter distributions}

Our NOAH-LSM model input parameter uncertainty domain is represented by a 5 -D hypercube (Table 1 ) with the distribution of each parameter being uniform (the norm for GLUE). It is defined as follows,

$X_{i} \sim U\left(p_{i}, q_{i}\right), i=1, \ldots \ldots, 5$,

where $p$ and $q$ form the lower and upper parameter ranges (column 1 of Table 1). Subscript $i$ refers to the specific parameter type (from 1 to 5 as listed in Table 1). $X$ represents the parameter value. These uniformly distributed parameters are then expressed as a series of a standard normal random variable $(s r v)$ as,

$x_{i, j}=p_{i}+\left(q_{i}-p_{i}\right)\left(\frac{1}{2}+\frac{1}{2} \operatorname{erf}\left(\varepsilon_{i}, j / \sqrt{2)}\right), i=1, \ldots, 5\right.$,

where $\varepsilon$ is a $s r v \sim \mathrm{N}(0,1)$ and $j$ denotes the index for a random realization. $\operatorname{erf}(x x)$ is the error function defined by the following integral,

$$
\operatorname{erf}(x x)=\frac{2}{\sqrt{\pi}} \int_{0}^{x x} e^{-w^{2}} d w .
$$

In Eq. (5), $x x$ is the $s r v$ and $w$ an intrinsic independent variable of the error function.

We have now expressed the random inputs (uniformly distributed model parameters) via srv's as $\{\varepsilon\}_{i=1}^{n}$ (where, $\mathrm{n}=$ 5). The choice of transforming the model parameters to the normal srvs is justified by mathematical tractability of functions of these srv's (Devroye, 1986).For example, other common univariate distributions such as gamma, exponential, Weibull, log-normal can all be transformed explicitly to normal srv's.

\subsection{Step two: polynomial chaos expansion}

Next, we represent our uncertain model output, $L$ - the likelihood measure (left-hand side of Eqs. 1 or 2), as an $n$-th order expansion of a Hermite Polynomial of $s r v$ 's. This step, called "Polynomial Chaos Expansion", follows from Ghanem and Spanos (1991). In this study we have considered second order expansion which is defined as follows,

$L_{2}=a_{0,2}+\sum_{i=1}^{n} a_{i, 2} \varepsilon_{i}+\sum_{i=1}^{n} a_{i i, 2}\left(\varepsilon_{i}^{2}-1\right)+\sum_{i=1}^{n-1} \sum_{j>1}^{n} a_{i j, 2} \varepsilon_{i} \varepsilon_{j}$

where the subscript after $L$ represents the order of the expansion.

5.3 Step three: calibration of coefficients of the Interpolator

From the above Eq. (5), it can be seen that the number of unknown coefficients (the $a$ 's in the right hand side) to be determined for second order polynomial chaos expansion are 21 . These unknown coefficients are now identified by generating the same number of model data points and solving the system of linear algebraic equations. Isukapalli and Georgopoulos (1999) provide guidelines on choosing model points for robust calibration of coefficients. The choice of the model points in this study is, however, left open to the user depending on the nature of the problem. We investigated this issue herein and report our findings in the next section. For calibration of polynomial coefficients we used the Singular Value Decomposition (SVD) (Press et al., 1999) because of its ability to handle ill-conditioned matrices (Press et al., 1999; Hossain and Anagnostou, 2004).

In Fig. 3 we summarize the algorithm for the interpolator. First, we generate a set of uniformly distributed model parameter sets from srvs (using Eqs. 3, 4 and Table 1). 21 points on the NOAH-LSM's parameter-output $(E)$ response surface are then chosen. The interpolator is then calibrated for its 21 coefficient values by solving the system of 21 linear algebraic equations. For a more global selection of calibration points, we derive 3 different sets of calibrated polynomials for the interpolators. The mean $E$ value predicted by the 3 calibrated interpolators is then defined as the most likely $E$ value for a sampled parameter set. The total number of different sets of calibration points required is considered subjective and depends on the nature of the sampling 

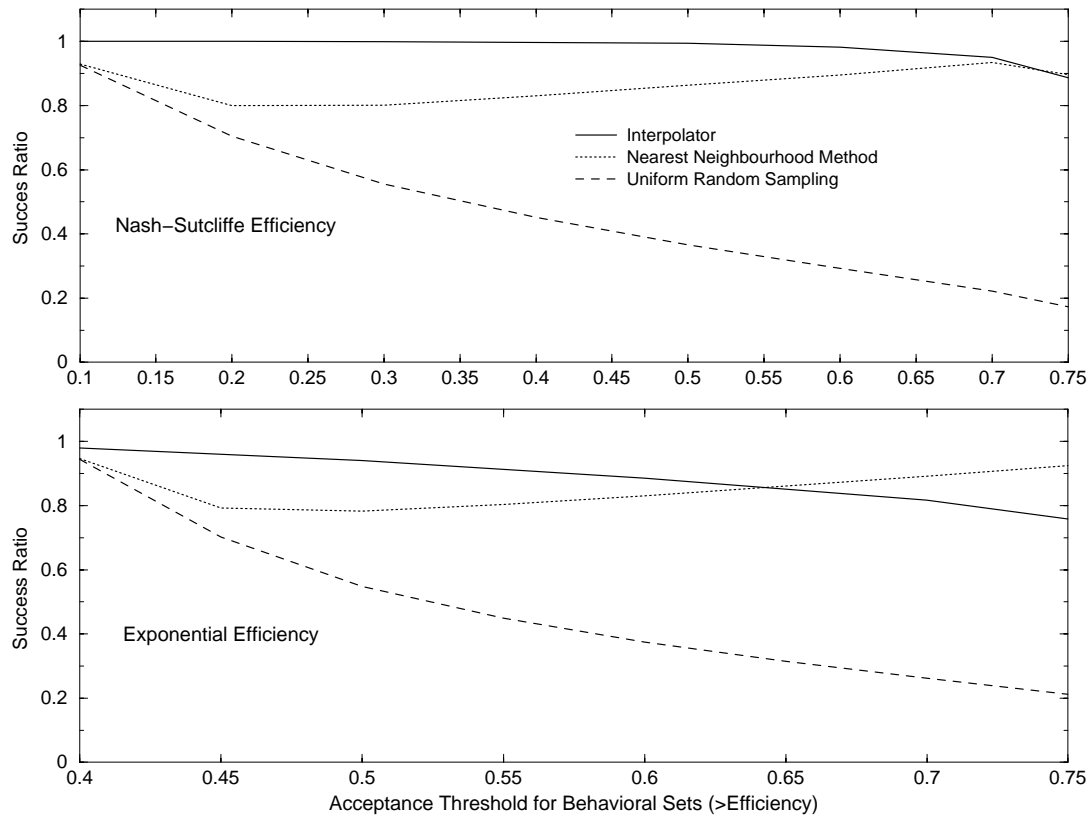

Fig. 4. General comparison of interpolator with Nearest-neighborhood $(N N)$ method and uniform random sampling as a predictor for sampled parameter sets in terms of Success Ratio.

problem. Herein we consider 3 sets as sufficient to yield effective results for a 5-D parameter hyperspace. Once the interpolator(s) is calibrated for NOAH-LSM on data available, we test its efficiency in parameter sampling in the following 4 steps: (i) sampling $\mathrm{N}(0,1)$ srvs; (ii) generating the corresponding family of uniformly distributed NOAH-LSM parameters from Eq. (4); (iii) computing the mean of the 3 interpolator-predicted $E$ values from Eq. (6); (iv) if the interpolator predicts a sampled parameter set to be behavioral, then testing its accuracy by actual execution of NOAH-LSM for that sampled parameter set. Note that the use of the interpolator in this fashion within the GLUE framework does not violate the fundamental requirement that parameters be sampled uniform distributions. It only helps to make an informed decision on sampling by providing an indication of whether the sampled parameter set is behavioral or non-behavioral before making the actual NOAH-LSM model run.

\section{Simulation framework}

The interpolator (which is now a simple algebraic equation) is potentially a 5-6 orders faster in computation than NOAHLSM and can therefore serve as a fast-running proxy for making Bayesian decisions on the degree of representativeness of sampled parameter sets for GLUE analysis. In almost all previous GLUE applications, behavioral and nonbehavioral parameter sets were identified through the actual time-consuming execution of the physically-complex model. This often resulted in high wastage of computational time as the majority of the runs were found to be non-behavioral (see Christaens and Feyen, 2002, for example). In this sim- ulation framework we tested the accuracy of the interpolator in stochastic modeling the parameter-output response surface for GLUE and assessed its potential in reducing the wastage of computational time due to the non-behavioral runs.

We conducted a total of 500000 NOAH-LSM simulations by sampling the same number of parameter sets randomly from the ranges in Table 1. This ensemble was further divided into 100 sub-divisions each containing 5000 parameter sets. Each of these sets had its respective "true" model response in terms of likelihood measures ( $E_{\mathrm{NS}}$ and $E_{\mathrm{EXP}}$ from Eqs. (1) and (2), respectively) determined from actual execution of NOAH-LSM. We then evaluated the sampling accuracy of the interpolator calibrated within each of these 100 sub-divisions to make generalizations on the mean and variability of its performance as a fast-running proxy. We first present a confusion matrix for sampled parameter sets below for the interpolator to define the performance measures whose description follows next.

Truth (from NOAH-LSM)

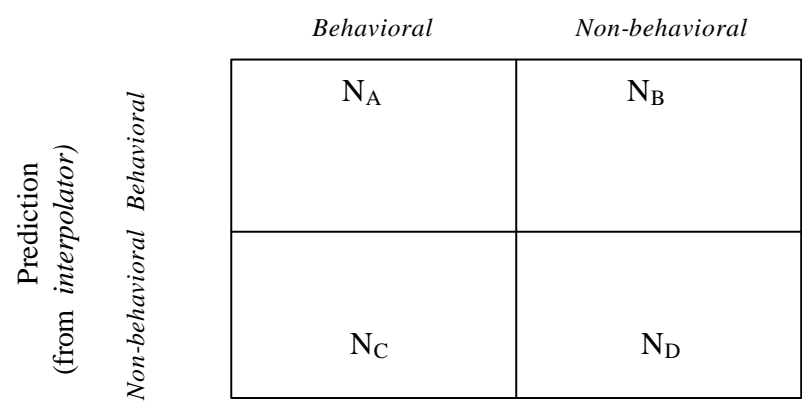


To define the probability of interpolator to successfully predict whether a sampled parameter set is behavioral or nonbehavioral (based on a given threshold for likelihood measure $L$ ) we define Success Ratio $(S R)$ as,

$S R=\frac{N_{A}}{N_{A}+N_{B}}$

The $S R$ indicates only a partial assessment of sampling efficiency. There can be instances where the interpolator is overly conservative in predicting a set as behavioral and thereby achieves a spuriously high $S R$ over very small samples of model executions. Thus, another measure, $(B S$, Eq. 8) was also defined. $B S$ quantifies the propensity of the interpolator to predict the behavioral sets as non-behavioral or missing regions of high likelihood values on the response surface.

$B S=\frac{N_{A}+N_{B}}{N_{A}+N_{C}}$

A $B S$ value of less than 1 would indicate that the interpolator has a tendency to be conservative in predicting correctly a sampled parameter set's likelihood value. A $B S$ value greater than 1 would indicate the interpolator's propensity to predict samples as behavioral. An ideal interpolator should therefore have a $B S$ of near 1.0 and $S R$ that is higher than that for uniform sampling.

Performance of the interpolator was compared with the fully uniform sampling of parameter sets using the above 2 measures (Eqs. 7 and 8). The Nearest-Neighborhood $(N N)$ search for interpolating parameter set's likelihood value was also compared herein (hereafter called $N N$ method). This type of sampling method was first introduced by Beven and Binley (1992) to address the computational concerns of the GLUE method. In the $N N$ method, a sampled point in parameter hyperspace is searched for the " $n$ " nearest neighboring points in a model's response surface that is preconstructed from a finite number sample points $(=1000$ preconstructed model points in this study). The probable likelihood value is then interpolated by the inverse squared distance technique. We have considered 6, 12 and 24 neighbors for the $N N$ method. A point to note is that the $N N$ method requires a computationally intensive sorting algorithm to rank all the distances from a sampled point in parameter hyperspace. The computing time for sorting increases as $N^{2}$ where $N$ is the size of the pre-constructed model points (Press et al., 1999). Hence a compromise is needed with the size of the pre-constructed model points when the dimension of the parameter hyperspace is high. This is considered a major weakness of the $N N$ method when compared to the interpolator.

\section{Results and discussion}

In Fig. 4 we show the mean $S R$ values of the 100 subdivisions (comprising the total 500000 sets) for the interpolator, $N N$ method (6 neighbors) and uniform random sampling for two different likelihood measures (Nash-Sutcliffe
Table 2. Mean Bias Score $(B S)$ values for the interpolator and $N N$ scheme.

\begin{tabular}{ccc}
\hline & Interpolator & $\begin{array}{c}\text { Nearest neighborhood } \\
\text { interpolator (6 neighbors) }\end{array}$ \\
\hline$>$ Threshold $E$ & & \\
(Nash-sutcliffe) & $B S$ & $B S$ \\
0.1 & 0.812 & 1.07 \\
0.2 & 0.858 & 1.21 \\
0.3 & 0.894 & 1.19 \\
0.4 & 0.808 & 1.09 \\
0.5 & 0.800 & 0.950 \\
0.6 & 0.867 & 0.700 \\
0.7 & 0.818 & 0.501 \\
\hline
\end{tabular}

efficiency - upper panel; Exponential efficiency - lower panel). Note that the $(1-S R)$ value actually represents the wastage of computational time due to non-behavioral runs of NOAH-LSM. This is because the sampled parameter sets were evaluated of their degree of representativeness by running the NOAH-LSM only after the prediction by the interpolator or the $N N$ method gave a strong indication of the set to be behavioral. The interpolator in Fig. 4 was calibrated with sample points that had a minimum $E$ value of 0.7 . We observe that the fully uniform random sampling can be very inefficient and result in high wastage of computational time (ranging from $50 \%-80 \%$ ) as the acceptance criterion for behavioral parameter sets increases $\left(E_{\mathrm{NS}}>0.4\right.$, upper panel; $E_{\mathrm{EXP}}>0.5$, lower panel). This observation justifies the wisdom of using a more efficient parameter sampling scheme for GLUE based on interpolation of the parameter response surface. The interpolator is able to demonstrate sampling efficiency in predicting correctly the nature of a sampled set (behavioral or non-behavioral?) even at high degrees of acceptance criterion. For Nash-Sutcliffe efficiency likelihood measure, the $S R$ value for interpolator is always found to be above 0.90 and about 0.10 higher than that of $N N$ method (Fig. 4, upper panel). The $S R$ value of the interpolator for Exponential efficiency likelihood measure appears to decrease moderately to 0.80 at the high acceptance criterion of $E_{\mathrm{EXP}}>0.60$ (lower panel, Fig. 4), and become less than that of the $N N$ method. However, for this case, the interpolator versus $N N$ method difference is found to be small (less than $15 \%$ ). Overall, when compared with the uniform random sampling, we note that the interpolator is able to reduce the wastage of computational time due to non-behavioral runs in the ranges of $10 \%-70 \%$.

Table 2 summarizes the mean values (of the 100 subdivisions of the 500000 sets) for $B S$ values for the interpolator and $N N$ method using the Nash-Sutcliffe efficiency as the likelihood measure. Similar statistics were observed for the Exponential efficiency likelihood measure, and is therefore not reported herein. We observe that the interpolator is moderately conservative $(B S<1.0)$ compared to the $N N$ method in accepting a sampled parameter set as behavioral. This is 

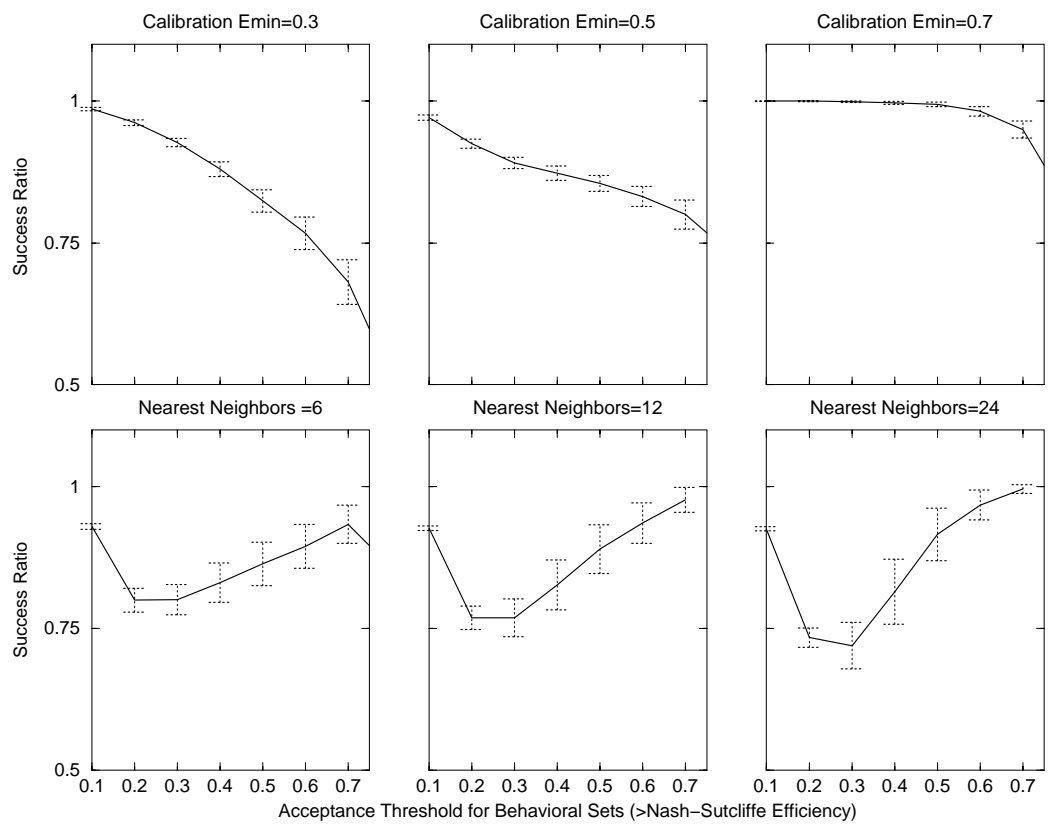

Fig. 5. (a) Impact of the choice of calibration points for interpolator (upper panel) and number of nearest neighbors in parameter search (lower panel) for Nash-Sutcliffe efficiency likelihood measure. The solid line indicates the mean of the 100 subdivisions (each containing 5000 NOAH-LSM simulations). One standard deviation of variability is indicated by the vertical error bars (dashed).
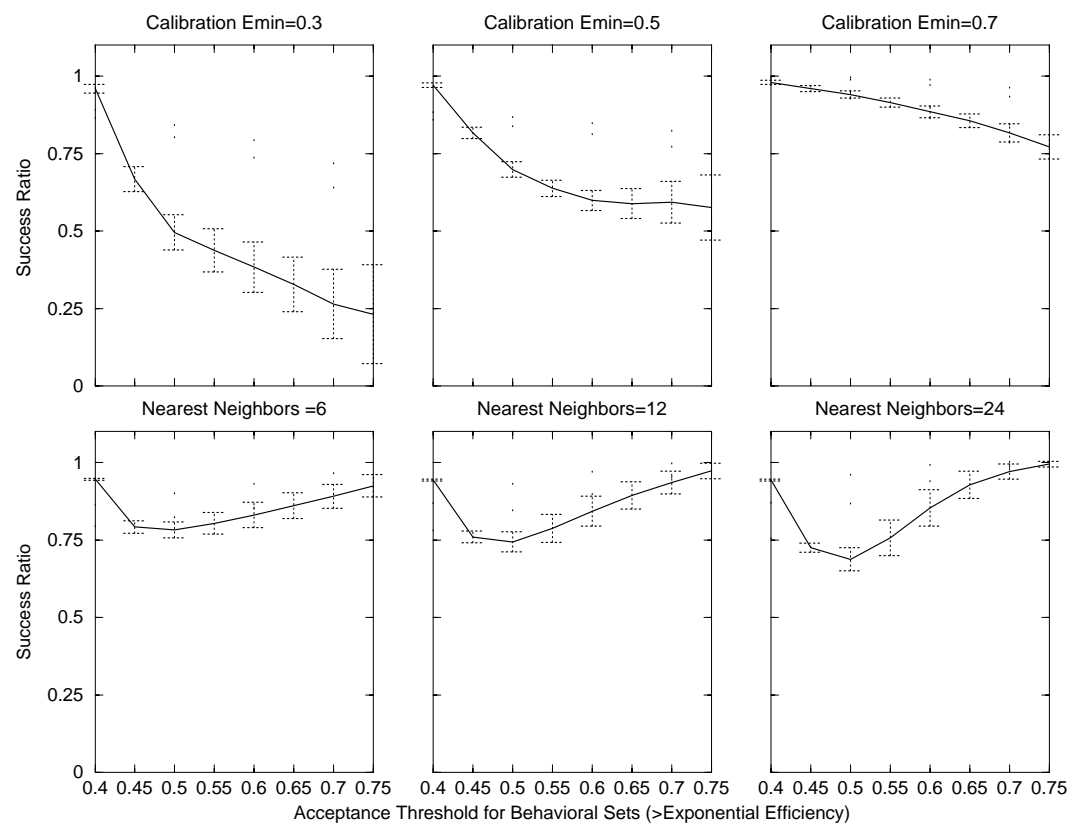

Fig. 5. (b) Same as in (a), but for exponential efficiency likelihood measure.

not necessarily considered a drawback of the interpolator as it can be executed as many times as needed to generate the desired sample size of behavioral parameter sets. The more qualifying aspect is whether the interpolator exhibits regions of local attractions in the response surface that are inconsistent with the uniform random sampling (discussed next).

In Figs. 5a and 5b, we explore certain calibration aspects of the interpolator and the $N N$ method for Nash-Sutcliffe and Exponential efficiency likelihood measures, respectively.
The upper panels show the effect of choice of calibration sample points for interpolator for three different criteria (selection of points based on a minimum Efficiency value of $0.3,0.5$ and 0.7). The lower panels show the effect of the " $n$ " - the number of nearest neighbors - in interpolating the likelihood value by the $N N$ method for 6,12 and 24 nearest neighbors. We observe that the choice of calibration points can have an impact on the efficiency ( $S R$ value) of the interpolator with the best performance achieved when the choice 

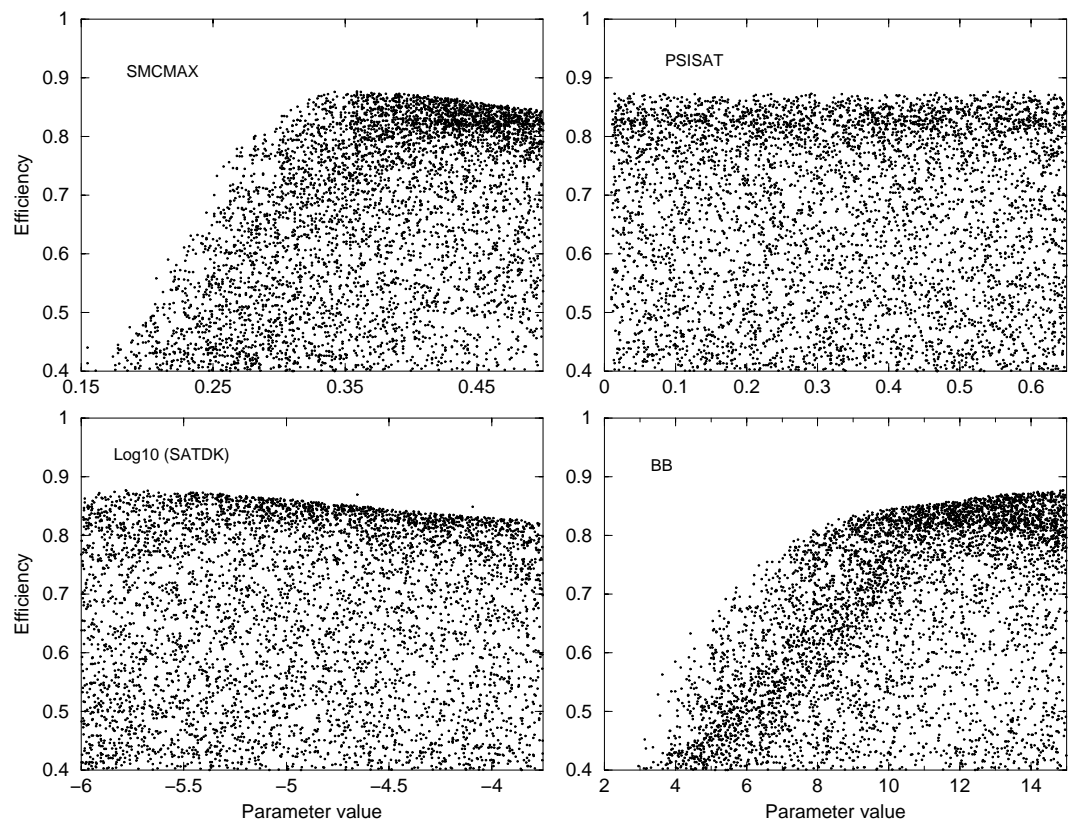

Fig. 6. (a) Dotty plots obtained from uniform random sampling of GLUE model parameters with Nash-Sutcliffe efficiency likelihood measure $>0.4$. The plots represent an ensemble of 5000 parameter sets.
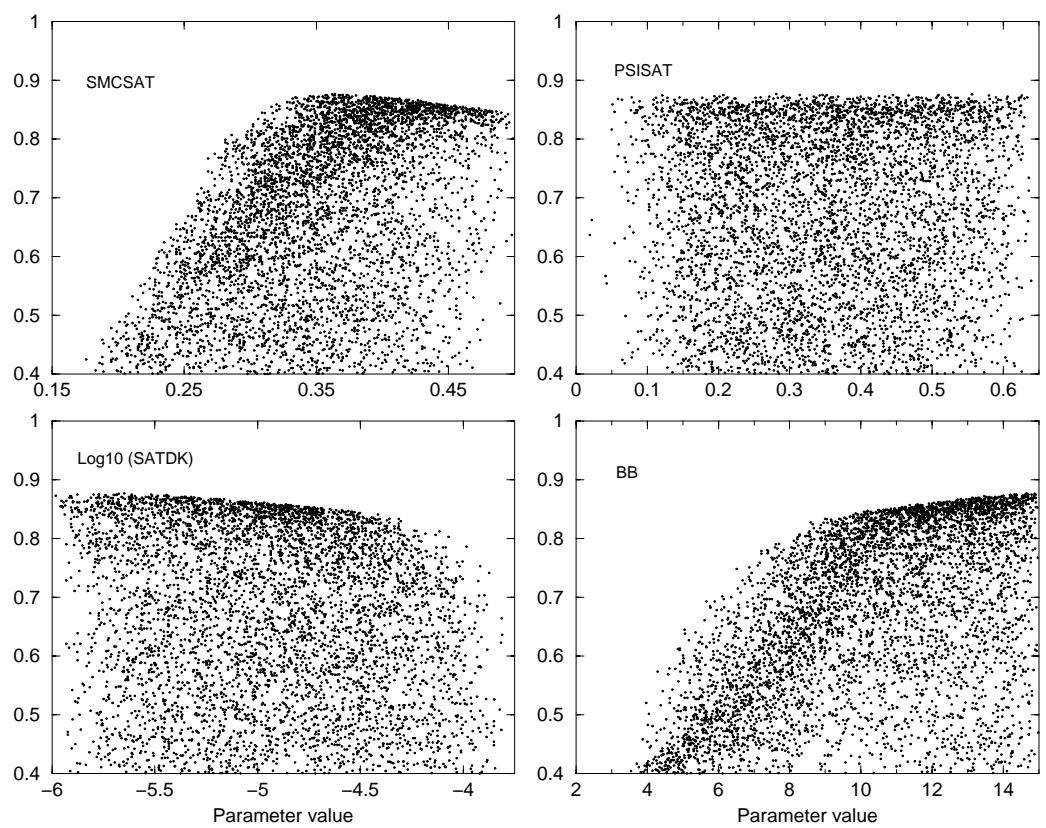

Fig. 6. (b) Same as in (a), but for the interpolator.

of points are highly behavioral (i.e. Emin $>0.7$ ). For $N N$ method, the choice of $n$ appears to have a negligible impact, although for both schemes, we observe that the variability in prediction increases as the acceptance criterion increases. Furthermore, the sampling efficiency (in terms of $S R$ ) of the $N N$ method appears to decrease in the moderate likelihood measure ranges $\left(0.2<E_{\mathrm{NS}}<0.5 ; 0.4<E_{\mathrm{EXP}}<0.6\right)$. We hypothesize that the simple inverse squared distance interpolation for $N N$ method is not universally effective for improved parameter sampling for LSMs because the response surface does not vary isotropically in a linear fashion with respect to parameters.

In Figs. 6a, b and c we compare the dotty plots obtained from the interpolator sampling and the random uniform sampling of GLUE model parameters. Dotty plots were first proposed by Beven and Binley (1992) as a simple way to demonstrate the parameter equifinality of a model. Against the likelihood value presented along the $y$-axis, the scatter of the pa- 

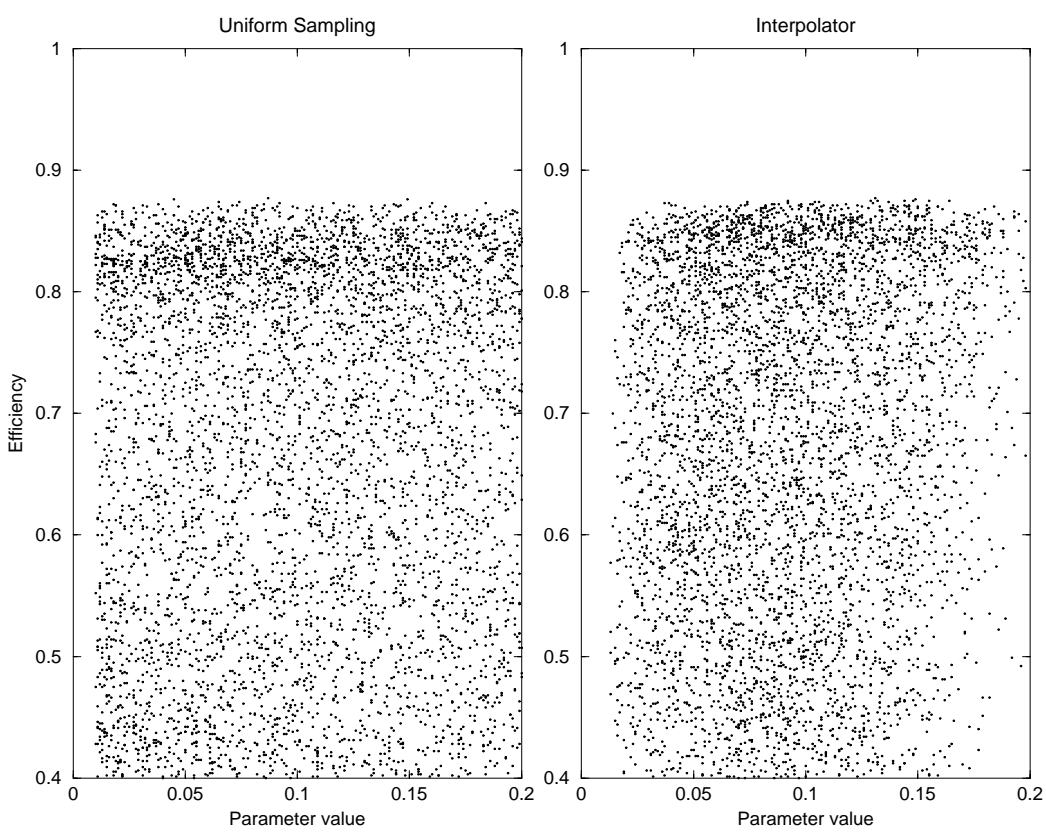

Fig. 6. (c) Same as Figs. 6a and 6b, but for the fifth NOAH-LSM parameter of SMCWLT.

rameters along the $\mathrm{x}$-axis is accepted as a qualitative measure of parameter equifinality. If the dotty plots derived from uniform random sampling are assumed as the reference, then the parameters sampled as behavioral via the initial screening of the interpolator should show similar scatter to represent consistent equifinality. This is an important aspect to assess for any parameter sampling scheme, which otherwise may render itself unsuitable for GLUE analysis. The dotty plots for the two likelihood measures were found to be similar. Hence we only show herein dotty plots pertaining to 5000 parameter sets sampled as behavioral for the Nash-Sutcliffe efficiency likelihood measure $E_{\mathrm{NS}}>0.4$. As seen by comparing Fig. 6b (interpolator dotty plots) with Fig. 6a (uniform random sampling) for the four NOAH-LSM parameters, we observe that the behavioral parameters sampled by interpolator represent, at least qualitatively, the same degree of equifinality as the reference (uniform) dotty plots. The fifth parameter comparison is shown in Fig. 6c (also found to be similar). The interpolator has no specific regions of local attraction of uneven sampling inconsistent with the uniform random sampling.

Finally, in Figs. 7a and 7b, we show a typical GLUE analysis with $90 \%$ confidence limits in soil moisture simulation uncertainty obtained from the aforementioned 5000 behavioral parameter sets - one ensemble sampled by uniform random sampling and the other ensemble sampled via the interpolator. The prediction quantiles produced by uniform random sampling are assumed as the reference for comparison here. For both likelihood measures (Nash-Sutcliffe efficiency likelihood measure - Fig. 7a lower panel; Exponential efficiency likelihood measure - Fig. 7b, lower panel) we observe negligible difference in the uncertainty estimation at the $90 \%$ confidence limits. However, a more quali- fying test for the preservation of the uncertainty structure in simulation is provided in Fig. 8 where we compare the Exceedance Probability $(E P)$ against the width of confidence limits (from $10 \%$ to $90 \%$ ). EP is defined as the number of times the observation (i.e. soil moisture measurement) is not enveloped by the predicted confidence limits normalized by the total number of timesteps in simulation. $E P$ would typically decrease monotonically with increasing width of the limits. A similarity of the monotonic decrease at high and low widths $(>80 \%$ and $<40 \%)$ is observed in Fig. 8. Since GLUE is typically used for uncertainty analyses at high confidence limits (Freer et al., 1996; Beven and Freer, 2001) this observation indicates that the interpolator is able to preserve sufficiently accurately the uncertainty structure of soil moisture simulation as would have been typically identified with random uniform sampling of the GLUE parameters. However, the use of the current formulation of the interpolator seems most accurate at high confidence limits ranging from $70 \%-90 \%$ for NOAH-LSM soil moisture simulations.

\section{Conclusions}

This study has presented a simple and efficient scheme for Bayesian assessment of uncertainty in soil moisture simulation by a Land Surface Model. The scheme was assessed within a MC simulation framework based on the GLUE methodology. Parameter sampling was improved in the proposed scheme by stochastic modeling of the parameter response surface that recognizes the inherent non-linear deterministic behavior of physically complex models. Uncertainty in soil moisture simulation was approximated through a polynomial chaos expansion of normal random variables that rep- 

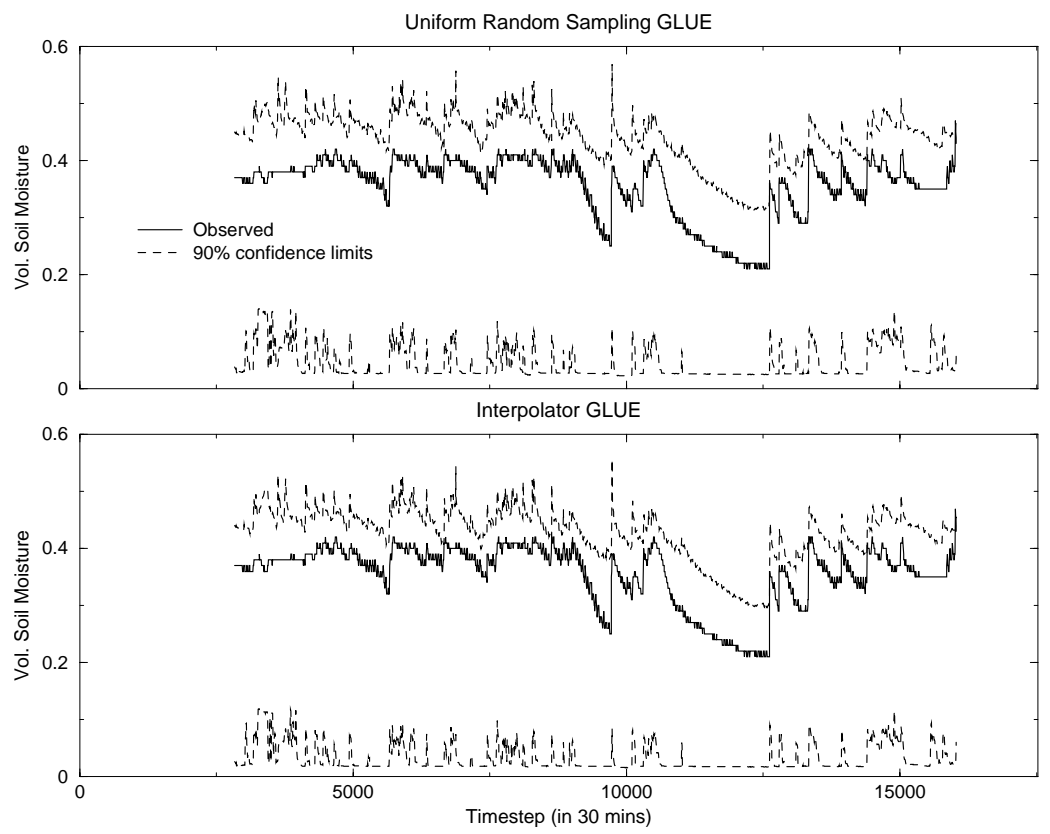

Fig. 7. (a) The GLUE uncertainty estimation of soil moisture simulation at $90 \%$ confidence limits for uniform random sampling (upper panel) and interpolator (lower panel). Nash-Sutcliffe efficiency likelihood measure $>0.4$ was used as the acceptance criterion for behavioral parameter sets. Uncertainty estimation for each scenario was conducted from the 5000 sampled sets shown in Figs. $6 \mathrm{a}, \mathrm{b}$ and $\mathrm{c}$.
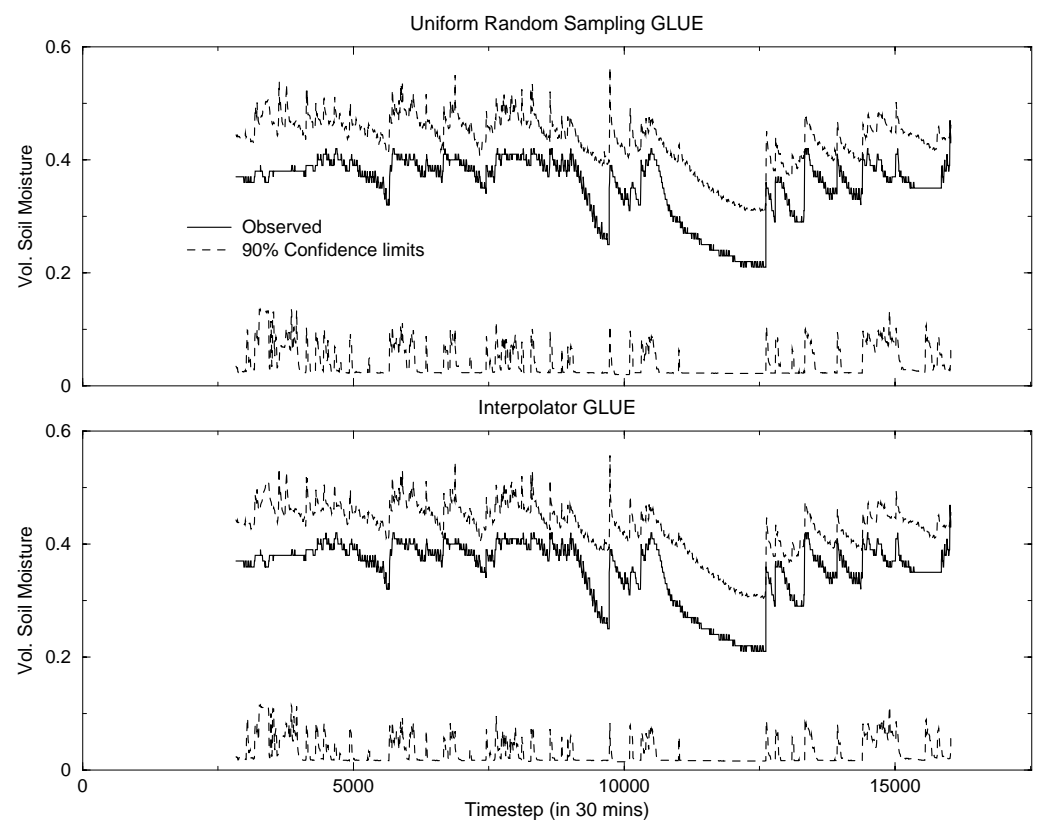

Fig. 7. (b) Same as (a), but for Exponential efficiency likelihood measure $>0.4$ as the acceptance criterion for behavioral parameter sets.

resented the model's parameter uncertainty. The calibrated polynomial (interpolator) was then used as a fast-running proxy to the slow-running model to predict the degree of representativeness of a randomly sampled model parameter set. The sampling scheme based on the interpolator was able to reduce computational burden of uniform random $\mathrm{MC}$ sampling for GLUE by about $10 \%-70 \%$. It was also found to be $10 \%$ more efficient and an order faster than the Nearestneighborhood sampling method. The GLUE based on the proposed sampling scheme preserved the uncertainty structure in soil moisture simulation at moderate to high confidence limits.

Because our proposed interpolator does not impose additional structural or distributional assumptions on GLUE method that could otherwise compromise its simplicity, it can readily apply to make GLUE parameter sampling for slowrunning models more efficient. Some of the natural extensions of this work include: (i) application of the interpolator 

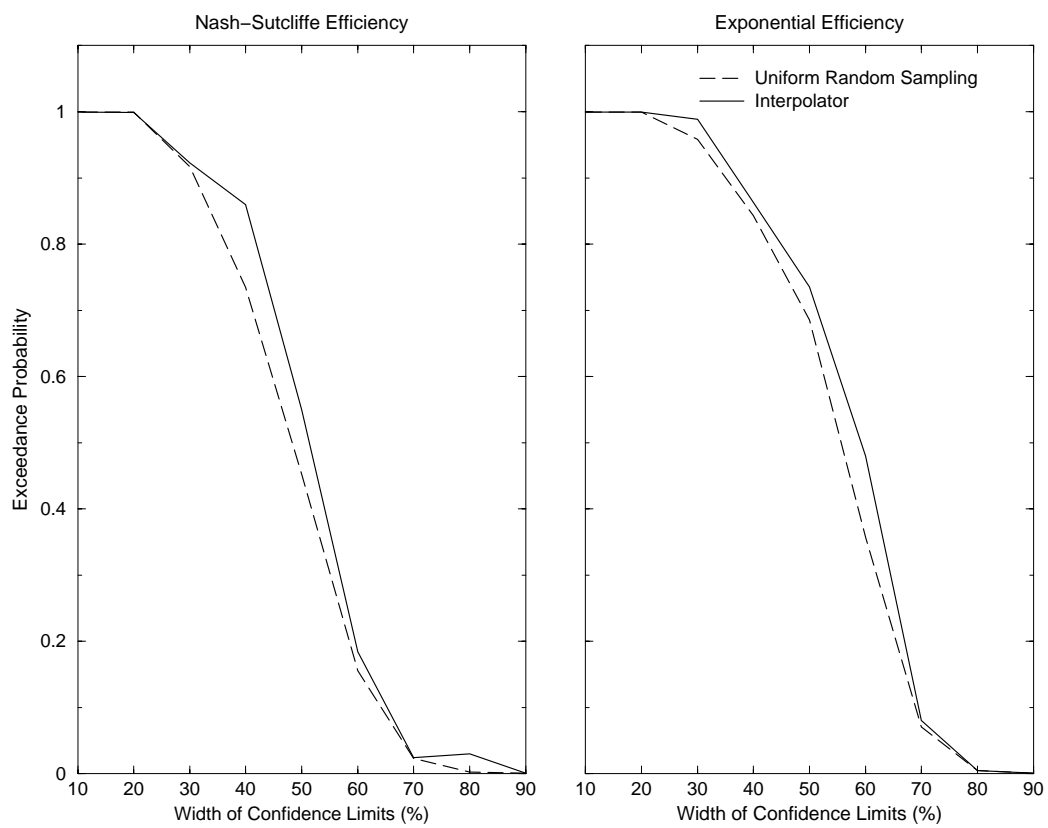

Fig. 8. Response of Exceedance Probability to width of predicted confidence limits. Left panel - Nash-Sutcliffe efficiency likelihood measure; Right panel - Exponential efficiency measure.

to other physically-complex models and hydrologic variables within the GLUE framework; (ii) investigating the conditions or assumptions that give rise to a chaotic and non-chaotic behavior in the hydrologic system and thereby attempt to connect the relationship of the hydrologic variable to the order of polynomial chaos expansions; and (iii) investigating the effect of the dimensional size of the parameter hyperspace on the sampling efficiency of the interpolator. It has also been suggested that the gradient information of the parameters with respect to model output, when assimilated in the polynomial chaos expansion, an increase in the prediction accuracy of the interpolator can be expected (Isukapalli and Georgopoulos, 1999). Another potential use of the stochastic non-linear response surface sampling scheme would be in applications to large-scale land surface simulations where model parameters are distributed as a matrix (2-D spatial domain) over large areal scales $\left(>10000 \mathrm{~km}^{2}\right)$ (note: in this study the parameters were a vector). For such applications, research is needed to explore convenient ways to mathematically reformulate the interpolator to handle such distributed parameters in spatial format. Work is on-going on some of the above aspects and we hope to report them in future.

Acknowledgements. The research associated with this paper was partially supported by the NASA New Investigator Program (Grant \#NAG5-8636). The first author was supported by a NASA Earth System Science Fellowship.

Edited by: B. Sivakumar

Reviewed by: two referees

\section{References}

Bates, B. C. and Campbell, E. P.: A Markov Chain Monte Carlo scheme for parameter estimation and inference in conceptual rainfall-runoff modeling, Water Resour. Res., 37, 3, 937-947, 2001.

Beck, M. B.: Water Quality Modeling: A Review of the Analysis of Uncertainty, Water Resour. Res., 23, 7, 1393-1442, 1987.

Beven, K. J. and Kirkby, M. J.: A physically-based variable contributing area model of basin hydrology. Hydrol. Sci. J., 24, 1, 43-69, 1979

Beven, K. J. and Freer, J.: Equifinality, data assimilation, and uncertainty estimation in mechanistic modeling of complex environmental systems using the GLUE methodology, J. of Hydrol., 249, 11-29, 2001.

Beven, K. J. and Binley, A.: The future of distributed models: Model calibration and uncertainty prediction, Hydrol. Proc., 6, 279-298, 1992.

Bras, R. L. and Rodriguez-Rodriguez-Iturbe, I.: Random Functions and Hydrology, Dover Publications, New York, 1993.

Clapp, R. B. and Hornberger, G. M.: Empirical equations for some soil hydraulic properties, Wat. Resour. Res., 14, 601-604, 1978.

Collins, D. C. and Avissar, R.: An Evaluation with the Fourier Amplitude Sensitivity Test (FAST) of which Land Surface Parameters are of Greatest Importance in Atmospheric modeling, J. of Climate, 7, 681-703, 1994.

Crawford, T. M., Stensrud, D. J., Mora, F., Merchant, J. W., and Wetzel, P. J.: Value of incorporating satellite-derived land cover data in MM5/PLACE for simulating surface temperatures, J. of Hydrometeorol., 2, 4, 453-468, 2001.

Christaens, K. and Feyen, J.: Constraining soil hydraulic parameter and output uncertainty of the distributed hydrological MIKE SHE model using the GLUE framework, Hydrol. Proc., 16, 2, 373, 2002.

Devroye, L.: Non-uniform random variate generation, SpringerVerlag, New York, 1986. 
Yongjiu Dai, Zeng, X., Dickinson, R. E., Baker, I., Bonan, G. B., Bosilovich, M. G., Denning, A. S., Dirmeyer, P. A., Houser, P. R., Keith, G. N., Oleson, W., Schlosser, C. A., and Yang, Z.-L.: The Common Land Model. Bull. Amer. Meteorol. Soc, August, 1013-1023, 2003.

Dickinson, R. E., Kennedy, P. J., and Wilson, M. F.: Biosphere Atmosphere Transfer Scheme (BATS) for the NCAR Community Climate Model, NCAR Tech. Note, NCAR TN275+STR, 69, 1986.

Franks, S. W., Gineste, P., Beven, K. J., and Merot P.: On constraining the predictions of a distributed model: The incorporation of fuzzy estimates of saturated areas into calibration process, Water Resour. Res., 34, 3, 787-797, 1998.

Franks, S. W. and Beven, K. J.: Bayesian estimation of uncertainty in land surface-atmosphere flux predictions, J. of Geophys. Res., 102, D20, 23 991-23 999, 1997.

Freer, J., Beven, K. J., and Ambroise, B.: Bayesian estimation of uncertainty in runoff prediction and the value of data: An application of the GLUE approach, Water Resour. Res., 32, 6, 21612173, 1996.

Gao, X., Sorooshian, S., and Gupta, H. V.: Sensitivity analysis of the biosphere-atmosphere transfer scheme, J. of Geophys. Res., 101, D3, 7279-7289, 1996.

Ghanem, R. and Spanos, P. D.: Stochastic Finite Elements: A Spectral Approach, Springer-Verlag, New York, 1991.

Gutman, G. and Ignatov, G. L.: Derivation of green vegetation fraction from NOAA/AVHRR for use in numerical weather prediction models, Int. J. Remote Sensing, 19, 7, 1533-1543, 1998.

Iman, R. L., Helton, J. C., and Campbell, J. C.: A approach to sensitivity analysis of Computer Models: Part I - Introduction, Input variable Selection and Preliminary Variable assessment, J. of Qual. Technol. , 13, 3, 174-183, 1981.

Henderson-Sellers, A.: A factorial assessment of sensitivity of the BATs Land-Surface Parameterization Scheme, J. of Climate, 6, 227-247, 1993.

Hossain, F., Anagnostou, E. N., Borga, M., and Dinku, T.: Hydrological Model Sensitivity to Parameter and Radar Rainfall Estimation Uncertainty. Hydrol. Proc., accepted, 2004.

Isukapalli, S. S. and Georgopolous, P. G.: Computational Methods for Efficient Sensitivity and Uncertainty Analysis of Models for Environmental and Biological Systems (Tech Rep CCL/EDMAS-03, Rutgers University), 1999.

Isukapalli, S. S., Roy A., and Georgopoulos, P. G.: Efficient sensitivity/uncertainty analysis using the combined stochastic response surface method and automated differentiation: Application to environmental and biological systems, Risk Analysis, 20, 4, 591-602, 2000.

Jayawardena A. W. and Lai, F.: Analysis and prediction of chaos in rainfall and streamflow time series, J. Hydrol., 153, 23-52, 1994.

Kremer, J. N.: Ecological Implications of parameter uncertainty in stochastic simulation, Ecological Modelling, 18, 187-207, 1983.

Krzysztofowicz, R.: Hydrologic uncertainty processor for probabilistic river stage forecasting, Water Resour. Res., 36, 11, 32653277, 2000.

Kuczera, G. and Parent, E.: Monte Carlo assessment of parameter uncertainty in conceptual catchment models: The Metropolis algorithm. J. of Hydrol., 211, 69-85, 1998.

Liu, K.: Soybeans: Chemistry, Technology and Utilization, Chapman and Hall: New York, 1-22, 1997.

Mahrt, L. and Ek, K.: The Influence of atmospheric stability on potential evaporation, J. Clim. Appl. Meteorol, 23, 1984.
McKay, M. D., Beckman, R. J., and Conover, W. J.: A Comparison of three methods for selecting values of input variables in the analysis of Output from a Computer Codes, Technometrics, 21, 2, 239-245, 1979.

Misirli, F., Gupta, H. V., Sorooshian, S., and Thiemann, M.: Bayesian Recursive estimation of Parameter and Output Uncertainty for watershed models, In Calibration of Watershed Models, edited by Duan, Q. J., Gupta, H. V., Sorooshian, S., Rousseau, A. N., and Turcotte, R., Water Science Application No. 6, AGU: Washington DC, 1125-1132, 2003.

Nash, J. E. and Sutcliffe, J. V.: River Flow forecasting through conceptual models, 1, A discussion of principles, J. Hydrol., 10, 282-290, 1970.

Norman, A. G.: Soybean Physiology, Agronomy, and Utilization, Academic Press: New York, 17-44, 1978.

Pan, H.-L. and Mahrt, L.: Interaction between soil hydrology and boundary layer development, Boundary Layer Meteorol, 38, 185-202, 1987.

Press, W. H., Teukolsky, S. A., Vetterling, W. T., and Flannery, B. P.: Numerical Recipes in Fortran 77 (Second Edition), Cambridge University Press, UK, 1999.

Rodriguez-Iturbe, R. I., Entekhabi, E., Lee, J. S., and Bras, R. L.: Non-linear dynamics of Soil Moisture at Climate Scales, 2. Chaotic Analysis, Water Resour. Res., 27, 7, 1991.

Romanowicz, R. and Beven, K.: Dynamic real-time prediction of flood inundation probabilities, Hydrological Sciences, 43, 2, 181-196, 1998.

Sellers, P. J., Mintz, Y., Sud, Y. C., and Dalcher, A.: A simple biosphere model ( $\mathrm{SiB}$ ) for use within general circulation models, J. of Atmos. Sci, 43, 505-531, 1986.

Sivakumar, B.: Chaos theory in hydrology: Important issues and interpretations, J. Hydrol., 227, 1-20, 2000.

Sivakumar, B., Berndtsson, R., Olsson, J., and Jinno, K.: Evidence of chaos in the rainfall-runoff process. Hydrological Sciences, 46, 1, 2001a.

Sivakumar, B., Sorooshian, S., Gupta V. J., and Gao, X.: A chaotic approach to rainfall disaggregation. Water Resour. Res., 37, 1, 61-72, 2001b.

Schulz, K. and Beven, K. J.: Data-supported robust parameterizations in land surface-atmosphere flux predictions: towards a topdown approach, Hydrol. Proc. 17, 2259-2277, 2003.

Schulz, K., Jarvis, A., and Beven, K.: The predictive uncertainty of land surface fluxes in response to increasing ambient carbon dioxide, J. of Climate, 14, 2551-2562, 2001.

Sridhar, V., Elliot, R. L., Chen F., and Botzge, J. A.: Validation of the NOAH-oSU land surface model using surface flux measurements in Oklahoma, J. of Geophys. Res., 107, D20, 2002.

Spear, R. C. and Hornberger, G. M.: Eutrophication in Peel Inlet, II, Identification of critical uncertainties via Generalized Sensitivity Analysis, Wat. Res., 4, 43-49, 1980.

Thiemann, M., Trosset, M., Gupta, H., and Sorooshian, S.: Bayesian recursive parameter estimation for hydrologic models, Water Resour. Res., 37, 10, 2521-2535, 2001.

Tyagi, A. and Haan, C. T.: Uncertainty analysis using first-order approximation method, Water Resour. Res., 37, 5, 1847-1858, 2001.

Young, P. C. and Beven, K. J.: Database mechanistic modeling and rainfall-flow non-linearity, Environmentrics, 5, 3, 335-363, 1994.

Wiener, N.: The Homogeneous Chaos, Amer. J. of Math., 60, 897893, 1938. 